\title{
A multiplicity result for a non-local parametric problem with periodic boundary conditions
}

\author{
Vincenzo Ambrosio, Rossella Bartolo and Giovanni Molica Bisci
}

\begin{abstract}
We look for bounded periodic solutions for a parametric fractional problem involving a continuous nonlinearity with subcritical growth. By using a variant of Caffarelli and Silvestre extension method adapted to the periodic case and variational tools we prove the existence of at least three bounded periodic solutions when the parameter varies in an appropriate range.
\end{abstract}

\section{Introduction}

In the present work we deal with the existence and the multiplicity of periodic solutions for the following nonlocal problem

$$
\begin{cases}\left(-\Delta+m^{2}\right)^{s} u=\lambda \alpha(x) f(u) & \text { in }(0, T)^{N} \\ u\left(x+T e_{i}\right)=u(x) & \text { for all } x \in \mathbb{R}^{N}, i=1, \ldots, N,\end{cases}
$$

where $T>0, m>0, s \in(0,1), N>2 s, \lambda$ is a positive real parameter, $\alpha: \mathbb{R}^{N} \rightarrow \mathbb{R}$ is a bounded periodic function, $\left(e_{i}\right)_{1 \leq i \leq N}$ is the canonical basis of $\mathbb{R}^{N}$ and $f: \mathbb{R} \rightarrow \mathbb{R}$ is a given function.

Due to their different and interesting applications joined to their challenging features from a mathematical viewpoint, nonlinear problems involving non-local operators have been widely studied by many authors (cf. [11], [14] and references therein).

In this paper we focus on a periodic non-local problem, taking advantage both of the approach firstly proposed in [9] and of recent papers [1], [2]. Namely, in [9] it is shown how to convert the original non-local problem into a local one in one more

Key words and phrases: fractional operators, periodic solutions, variational methods, multiple solutions.

2010 Mathematics Subject Classification: primary 49J35; secondary 35A15, 35S15, 47G20. 
dimension by means of a Dirichlet to Neumann map. On the other hand, in [1], [2] such method has been carefully written in the periodic case. We refer the reader to Section 2 for a detailed description of the extension periodic method. Then we use some classical tools in critical point theory in order to find multiple solutions of the new elliptic problem and finally go back to weak solutions of the original one. See, for instance, the papers [3], [4], [6]-[8] and [12] for related topics.

Here the nonlocal operator $\left(-\Delta+m^{2}\right)^{s}$ is defined by a spectral decomposition, by using the powers of the eigenvalues of $-\Delta+m^{2}$ with periodic boundary conditions. Let $u \in \mathcal{C}_{T}^{\infty}\left(\mathbb{R}^{N}\right)$, that is $u$ is infinitely differentiable in $\mathbb{R}^{N}$ and $T$-periodic in each variable. Then $u$ can be written as a Fourier series:

$$
u(x)=\sum_{k \in \mathbb{Z}^{N}} b_{k} \frac{e^{\imath \omega k \cdot x}}{\sqrt{T^{N}}} \quad\left(x \in \mathbb{R}^{N}\right)
$$

where

$$
\omega:=\frac{2 \pi}{T} \quad \text { and } \quad b_{k}:=\frac{1}{\sqrt{T^{N}}} \int_{(0, T)^{N}} u(x) e^{-\imath \omega k \cdot x} d x \quad\left(k \in \mathbb{Z}^{N}\right)
$$

are the Fourier coefficients of $u$.

Hence, the operator $\left(-\Delta+m^{2}\right)^{s}$ is given by

$$
\left(-\Delta+m^{2}\right)^{s} u:=\sum_{k \in \mathbb{Z}^{N}} b_{k}\left(\omega^{2}|k|^{2}+m^{2}\right)^{s} \frac{e^{\imath \omega k \cdot x}}{\sqrt{T^{N}}} .
$$

Moreover, if $u:=\sum_{k \in \mathbb{Z}^{N}} b_{k} \frac{e^{\imath \omega k \cdot x}}{\sqrt{T^{N}}}$ and $v:=\sum_{k \in \mathbb{Z}^{N}} d_{k} \frac{e^{\imath \omega k \cdot x}}{\sqrt{T^{N}}}$, we have that the quadratic form

$$
\mathcal{Q}(u, v):=\sum_{k \in \mathbb{Z}^{N}}\left(\omega^{2}|k|^{2}+m^{2}\right)^{s} b_{k} \bar{d}_{k}
$$

can be extended by density on the Hilbert space

$$
\mathbb{H}_{T}^{s}:=\left\{u=\sum_{k \in \mathbb{Z}^{N}} b_{k} \frac{e^{\imath \omega k \cdot x}}{\sqrt{T^{N}}} \in L^{2}(0, T)^{N}: \sum_{k \in \mathbb{Z}^{N}}\left(\omega^{2}|k|^{2}+m^{2}\right)^{s}\left|b_{k}\right|^{2}<+\infty\right\}
$$

endowed with the norm

$$
|u|_{\mathbb{H}_{T}^{s}}:=\left(\sum_{k \in \mathbb{Z}^{N}}\left(\omega^{2}|k|^{2}+m^{2}\right)^{s}\left|b_{k}\right|^{2}\right)^{1 / 2}
$$

From a physical point of view, when $s=1 / 2$, the operator $\left(-\Delta+m^{2}\right)^{\frac{1}{2}}$ corresponds to the Hamiltonian of a free relativistic particle of mass $m$ (cf. [13]). On the other hand, $\left(-\Delta+m^{2}\right)^{s}-m^{2 s}$ plays an important role in Stochastic Processes 
Theory, because it is an infinitesimal generator of a Lévy process $\left\{X_{t}^{m}\right\}_{t \geq 0}$ called the relativistic $2 s$-stable process; for more details we refer to [10] and [18].

We are able to find a bounded interval of positive parameters $\lambda$ for which the corresponding problem $\left(P_{\lambda}\right)$ admits at least three $L^{\infty}$-bounded weak solutions in an appropriate Sobolev space. Once we have written problem $\left(P_{\lambda}\right)$ as a local one using the notion of harmonic extension and the Dirichlet-to-Neumann map in the periodic setting (cf. Section 2), we study the existence of critical points of the energy functional associated to the problem (cf. (2.6)). Namely, a local minimum result for smooth functionals (cf. [17] and, here, Theorem 2.6) and a classic minimization argument give us the existence of two (distinct) critical points, therefore by [15, Theorem 4] it follows the existence of a third one. Finally, the traces of such solutions give us back three solutions to $\left(P_{\lambda}\right)$ which are also bounded.

For the reader's convenience, we list some notations at the end of this section.

Our main result can be stated as follows.

Theorem 1.1. Let $\alpha: \mathbb{R}^{N} \rightarrow \mathbb{R}$ be a T-periodic and $L^{\infty}$-map satisfying

$$
\alpha_{0}:=\operatorname{essinf}_{x \in(0, T)^{N}} \alpha(x)>0 .
$$

Let $f: \mathbb{R} \rightarrow \mathbb{R}$ be a continuous function such that

there exist two positive constants $a_{1}, a_{2}$ and $q \in\left[1,2_{s}^{*}\right)$ such that

$$
|f(t)| \leq a_{1}+a_{2}|t|^{q-1} \quad \text { for all } t \in \mathbb{R},
$$

and the potential $F(t):=\int_{0}^{t} f(r) d r$ satisfies the sign-condition

$$
\inf _{t \in[0,+\infty)} F(t) \geq 0
$$

Moreover, we assume that the following algebraic inequality holds

$$
\frac{F(\varrho)}{\varrho^{2}}>\frac{m^{2 s}}{2}\left(a_{1} \frac{\beta_{1}}{\gamma}+a_{2} \beta_{2} \gamma^{q-2}\right)
$$

for some $\varrho, \gamma>0$ satisfying

$$
\varrho>\sqrt{\frac{2}{\varkappa_{s}}} \frac{\gamma}{m^{s} T^{N / 2}},
$$

where

$$
\beta_{1}:=\frac{\varkappa_{s} \sqrt{2} c_{1}|\alpha|_{L^{\infty}(0, T)^{N}}}{\alpha_{0}}, \quad \beta_{2}:=\frac{\varkappa_{s} 2^{\frac{q}{2}} c_{q}^{q}|\alpha|_{L^{\infty}(0, T)^{N}}}{q \alpha_{0}}
$$

and $c_{1}, c_{q}$ are as in (2.2) below. 
In addition, we suppose that there exist $M>0$ and $\delta \in(0,2)$ such that

$$
F(t) \leq M\left(1+|t|^{\delta}\right) \quad \text { for all } t \in \mathbb{R} .
$$

Then, taking $\mu_{1}, \mu_{2}$ respectively as in (3.10) and (3.3), for each $\lambda \in\left(\mu_{1}, \mu_{2}\right)$, problem $\left(P_{\lambda}\right)$ has at least three weak solutions $u_{1}^{\lambda}, u_{2}^{\lambda}, u_{3}^{\lambda} \in L^{\infty}(0, T)^{N} \cap \mathbb{H}_{T}^{s}$.

This paper is organized as follows: in Section 2 we recall some preliminaries about fractional periodic Sobolev spaces and we recall the extension method, besides some well known critical point theorems. Then in Section 3 we prove our main result.

\section{Notations}

- $\left(X,\|\cdot\|_{X}\right)$ denotes a Banach space, $\left(X^{\prime},\|\cdot\|_{X^{\prime}}\right)$ its topological dual;

- $|\cdot|_{L^{r}(0, T)^{N}}$ the usual norm in the Lebesgue space $L^{r}(0, T)^{N}, 1 \leq r \leq+\infty$;

- $2_{s}^{*}:=\frac{2 N}{N-2 s}$ the critical exponent for Sobolev embeddings;

- if $\Omega$ is a domain of $\mathbb{R}^{N}, L^{2}\left(\Omega \times \mathbb{R}_{+}, y^{1-2 s}\right)$ is the space of all measurable functions $v$ on $\Omega \times \mathbb{R}_{+}$such that

$$
\iint_{\Omega \times \mathbb{R}_{+}} y^{1-2 s} v^{2} d x d y<+\infty
$$

- $H_{m}^{1}\left(\Omega \times \mathbb{R}_{+}, y^{1-2 s}\right)$ is the space of all $v$ such that $v, \nabla v \in L^{2}\left(\Omega \times \mathbb{R}_{+}, y^{1-2 s}\right)$ with square norm

$$
\begin{aligned}
& \qquad \int_{\Omega \times \mathbb{R}_{+}} y^{1-2 s}\left(|\nabla v|^{2}+m^{2} v^{2}\right) d x d y ; \\
& \text { - } \varkappa_{s}:=2^{1-2 s} \frac{\Gamma(1-s)}{\Gamma(s)} \text {. }
\end{aligned}
$$

\section{Preliminaries and functional setting}

As announced in Section 1 we realize the operator $\left(-\Delta+m^{2}\right)^{s}$ with periodic boundary condition as a Dirichlet to Neumann map.

Firstly we recall some preliminary results which will be used throughout the paper. Starting from papers [1], [2], we collect basic notions about fractional periodic Sobolev spaces.

Let us denote by

$$
\mathbb{R}_{+}^{N+1}:=\left\{(x, y) \in \mathbb{R}^{N+1}: x \in \mathbb{R}^{N}, y>0\right\}
$$

the upper half-space in $\mathbb{R}^{N+1}$; moreover we define the half-cylinder in $\mathbb{R}_{+}^{N+1}, \mathcal{S}_{T}:=$ $(0, T)^{N} \times(0, \infty)$, its basis $\partial^{0} \mathcal{S}_{T}:=(0, T)^{N} \times\{0\}$ and its lateral boundary $\partial_{L} \mathcal{S}_{T}:=$ $\partial(0, T)^{N} \times[0,+\infty)$. 
Let $\mathcal{C}_{T}^{\infty}\left(\mathbb{R}^{N}\right)$ be the space of functions $u \in \mathcal{C}^{\infty}\left(\mathbb{R}^{N}\right)$ such that $u$ is $T$-periodic in each variable, that is

$$
u\left(x+T e_{i}\right)=u(x) \quad \text { for all } x \in \mathbb{R}^{N}, i=1, \ldots, N .
$$

As recalled in Section 1, the fractional Sobolev space $\mathbb{H}_{T}^{s}$ is defined as the closure of $\mathcal{C}_{T}^{\infty}\left(\mathbb{R}^{N}\right)$ with respect to the norm

$$
|u|_{\mathbb{H}_{T}^{s}}:=\sqrt{\sum_{k \in \mathbb{Z}^{N}}\left(\omega^{2}|k|^{2}+m^{2}\right)^{s}\left|b_{k}\right|^{2}},
$$

where $b_{k}:=\frac{1}{\sqrt{T^{N}}} \int_{(0, T)^{N}} u(x) e^{-\imath \omega k \cdot x} d x$ are the Fourier coefficients of $u$. Furthermore, we define the functional space $\mathbb{X}_{T}^{s}$ as the completion of

$$
\begin{array}{r}
\mathcal{C}_{T}^{\infty}\left(\overline{\mathbb{R}_{+}^{N+1}}\right):=\left\{v \in \mathcal{C}^{\infty}\left(\overline{\mathbb{R}_{+}^{N+1}}\right): v\left(x+T e_{i}, y\right)=v(x, y)\right. \\
\left.\quad \text { for every }(x, y) \in \overline{\mathbb{R}_{+}^{N+1}}, i=1, \ldots, N\right\}
\end{array}
$$

under the $H^{1}\left(\mathcal{S}_{T}, y^{1-2 s}\right)$-norm

$$
\|v\|_{\mathbb{X}_{T}^{s}}:=\sqrt{\iint_{\mathcal{S}_{T}} y^{1-2 s}\left(|\nabla v|^{2}+m^{2} v^{2}\right) d x d y} .
$$

Now, we state a result related to the existence of a trace operator between the spaces $\mathbb{X}_{T}^{s}$ and $\mathbb{H}_{T}^{s}$ (cf. [1, Theorem 3] for the proof).

Theorem 2.1. There exists a surjective linear operator $\operatorname{Tr}: \mathbb{X}_{T}^{s} \rightarrow \mathbb{H}_{T}^{s}$ such that

(i) $\operatorname{Tr}(v)=\left.v\right|_{\partial^{0} \mathcal{S}_{T}}$ for all $v \in \mathcal{C}_{T}^{\infty}\left(\overline{\mathbb{R}_{+}^{N+1}}\right) \cap \mathbb{X}_{T}^{s}$.

(ii) $\operatorname{Tr}$ is bounded and

$$
\sqrt{\varkappa_{s}}|\operatorname{Tr}(v)|_{\mathbb{H}_{T}^{s}} \leq\|v\|_{\mathbb{X}_{T}^{s}} \quad \text { for all } v \in \mathbb{X}_{T}^{s}
$$

In particular, the inequality in (2.1) is an equality for some $v \in \mathbb{X}_{T}^{s}$ if and only if $v$ weakly solves the equation

$$
-\operatorname{div}\left(y^{1-2 s} \nabla v\right)+m^{2} y^{1-2 s} v=0 \quad \text { in } \mathcal{S}_{T} .
$$

The following crucial embedding results have been proved in [1, Theorem 4].

Theorem 2.2. Let $N>2 s$. Then $\operatorname{Tr}\left(\mathbb{X}_{T}^{s}\right)$ is continuously embedded in $L^{q}(0, T)^{N}$ for all $1 \leq q \leq 2_{s}^{*}$, that is there exists $c_{q}>0$ such that

$$
|\operatorname{Tr}(v)|_{L^{q}(0, T)^{N}} \leq c_{q}\|v\|_{\mathbb{X}_{T}^{s}} \quad \text { for all } v \in \mathbb{X}_{T}^{s} .
$$

Moreover, $\operatorname{Tr}\left(\mathbb{X}_{T}^{s}\right)$ is compactly embedded in $L^{q}(0, T)^{N}$ for any $1 \leq q<2_{s}^{*}$. 
Theorems 2.1 and 2.2 allow us to introduce the notion of extension for a function $u \in \mathbb{H}_{T}^{s}$.

Theorem 2.3. Let $u \in \mathbb{H}_{T}^{s}$. Then, there exists a unique $v \in \mathbb{X}_{T}^{s}$ such that

$$
\begin{cases}-\operatorname{div}\left(y^{1-2 s} \nabla v\right)+m^{2} y^{1-2 s} v=0 & \text { in } \mathcal{S}_{T} \\ v_{\mid\left\{x_{i}=0\right\}}=v_{\mid\left\{x_{i}=T\right\}} & \text { on } \partial_{L} \mathcal{S}_{T} \\ v(\cdot, 0)=u & \text { on } \partial^{0} \mathcal{S}_{T}\end{cases}
$$

and

$$
-\lim _{y \rightarrow 0^{+}} y^{1-2 s} \frac{\partial v}{\partial y}(x, y)=\varkappa_{s}\left(-\Delta+m^{2}\right)^{s} u(x) \quad \text { in } \mathbb{H}_{T}^{-s}
$$

where the boundary condition on $\partial^{0} \mathcal{S}_{T}$ is in the sense of trace, $\mathbb{H}_{T}^{-s}$ is the dual space of $\mathbb{H}_{T}^{s}$ and the notation $v_{\mid\left\{x_{i}=0\right\}}=v_{\mid\left\{x_{i}=T\right\}}$ on $\partial_{L} \mathcal{S}_{T}$ means

$$
v\left(x_{1}, \ldots, x_{i-1}, 0, x_{i+1}, \ldots, x_{N}, y\right)=v\left(x_{1}, \ldots, x_{i-1}, T, x_{i+1}, \ldots, x_{N}, y\right)
$$

for all $i \in\{1, \ldots, N\}, y \geq 0$. We say that $v \in \mathbb{X}_{T}^{s}$ is the extension of $u \in \mathbb{H}_{T}^{s}$.

In particular, if $u=\sum_{k \in \mathbb{Z}^{N}} b_{k} \frac{e^{\imath \omega k \cdot x}}{\sqrt{T^{N}}}$, then $v$ is given by

$$
v(x, y)=\sum_{k \in \mathbb{Z}^{N}} b_{k} \theta_{k}(y) \frac{e^{\imath \omega k \cdot x}}{\sqrt{T^{N}}}
$$

where $\theta_{k}(y):=\theta\left(\sqrt{\omega^{2}|k|^{2}+m^{2}} y\right)$ and $\theta(y) \in H^{1}\left(\mathbb{R}_{+}, y^{1-2 s}\right)$ solves the following ODE

$$
\left\{\begin{array}{l}
\theta^{\prime \prime}+\frac{1-2 s}{y} \theta^{\prime}-\theta=0 \quad \text { in } \mathbb{R}_{+} \\
\theta(0)=1, \quad \theta(+\infty)=0 .
\end{array}\right.
$$

Let us observe that

$$
\theta(y)=\frac{2}{\Gamma(s)}\left(\frac{y}{2}\right)^{s} K_{s}(y)
$$

where $K_{s}$ denotes the modified Bessel function of the second kind with order $s$.

Moreover, $v$ satisfies the properties

(i) $v$ is smooth for $y>0$ and $T$-periodic in $x$;

(ii) $\|v\|_{\mathbb{X}_{T}^{s}} \leq\|z\|_{\mathbb{X}_{T}^{s}}$ for any $z \in \mathbb{X}_{T}^{s}$ such that $\operatorname{Tr}(z)=u$;

(iii) $\|v\|_{\mathbb{X}_{T}^{s}}=\sqrt{\varkappa_{s}}|u|_{\mathbb{H}_{T}^{s}}$. 
By using the extension method in periodic setting, the study of $\left(P_{\lambda}\right)$ is then equivalent to study the solutions $v \in \mathbb{X}_{T}^{s}$ of the following problem

$$
\begin{cases}-\operatorname{div}\left(y^{1-2 s} \nabla v\right)+m^{2} y^{1-2 s} v=0 & \text { in } \mathcal{S}_{T} \\ v_{\mid\left\{x_{i}=0\right\}}=v_{\mid\left\{x_{i}=T\right\}} & \text { on } \partial_{L} \mathcal{S}_{T} \\ \partial_{\nu}^{1-2 s} v=\varkappa_{s} \lambda \alpha(x) f(v) & \text { on } \partial^{0} \mathcal{S}_{T}\end{cases}
$$

where

$$
\partial_{\nu}^{1-2 s} v:=-\lim _{y \rightarrow 0^{+}} y^{1-2 s} \frac{\partial v}{\partial y}(x, y)
$$

is the conormal exterior derivative of $v$.

More precisely, we can reformulate the nonlocal problem $\left(P_{\lambda}\right)$ with periodic boundary conditions in a local way according to the following definitions.

Definition 2.4. Fixing $\lambda>0$, we say that $v \in \mathbb{X}_{T}^{s}$ is a weak solution to (2.4) if

$$
\iint_{\mathcal{S}_{T}} y^{1-2 s}\left(\nabla v \nabla \varphi+m^{2} v \varphi\right) d x d y=\varkappa_{s} \lambda \int_{\partial^{0} \mathcal{S}_{T}} \alpha(x) \operatorname{Tr}(v) \operatorname{Tr}(\varphi) d x
$$

for every $\varphi \in \mathbb{X}_{T}^{s}$.

We can also give the notion of weak solution to problem (2.4) as follows.

Definition 2.5. Fixing $\lambda>0$, we say that $u \in \mathbb{H}_{T}^{s}$ is a weak solution to $\left(P_{\lambda}\right)$ if $u=\operatorname{Tr}(v)$ and $v \in \mathbb{X}_{T}^{s}$ is a weak solution to (2.4) according to Definition 2.4.

Therefore, in order to find weak solutions to (2.4), we introduce the energy functional $\mathcal{E}_{\lambda}: \mathbb{X}_{T}^{s} \rightarrow \mathbb{R}$ defined by

$$
\mathcal{E}_{\lambda}(v):=\frac{1}{2} \iint_{\mathcal{S}_{T}} y^{1-2 s}\left(|\nabla v|^{2}+m^{2} v^{2}\right) d x d y-\varkappa_{s} \lambda \int_{\partial^{0} \mathcal{S}_{T}} \alpha(x) F(\operatorname{Tr}(v)) d x,
$$

for every $v \in \mathbb{X}_{T}^{s}$.

By (1.2), it is straightforward to prove that $\mathcal{E}_{\lambda}$ is well-defined and of class $C^{1}$ in $\mathbb{X}_{T}^{s}$. In Section 3 we prove the existence of weak solutions to (2.4) by suitable variational methods.

Firstly, we recall the following abstract theorem due to Ricceri (cf. [17]), restated here in a more convenient form.

Theorem 2.6. Let $X$ be a reflexive real Banach space and $\Phi, \Psi: X \rightarrow \mathbb{R}$ be two Gâteaux differentiable functionals such that $\Phi$ is strongly continuous, sequentially weakly lower semicontinuous and coercive. Furthermore, assume that $\Psi$ is sequentially weakly upper semicontinuous. Setting for every $r>\inf _{X} \Phi$

$$
\varphi(r):=\inf _{w \in \Phi^{-1}((-\infty, r))} \frac{\left(\sup _{z \in \Phi^{-1}((-\infty, r))} \Psi(z)\right)-\Psi(w)}{r-\Phi(w)},
$$


then for each $r>\inf _{X} \Phi$ and $\lambda \in(0,1 / \varphi(r))$, the restriction of

$$
\mathcal{F}_{\lambda}:=\Phi-\lambda \Psi
$$

to $\Phi^{-1}((-\infty, r))$ admits a global minimum, which is a critical point (local minimum) of $\mathcal{F}_{\lambda}$ in $X$.

Moreover, applying the following classical theorem by P. Pucci and J. Serrin (cf. [15, Theorem 4] and [16, Theorem 3.10]), we will deduce the existence of a further critical point. Before stating the result, we recall the well known definition of the Palais-Smale condition: a $C^{1}$-functional $\mathcal{F}: X \rightarrow \mathbb{R}$ satisfies the Palais-Smale condition if for all $c \in \mathbb{R}$

every sequence $\left(v_{n}\right)_{n} \subset X$ such that

$$
\mathcal{F}\left(v_{n}\right) \longrightarrow c \text { and }\left\|\mathcal{F}^{\prime}\left(v_{n}\right)\right\|_{X^{\prime}} \longrightarrow 0 \quad \text { as } n \longrightarrow+\infty
$$

admits a convergent subsequence in $X$.

Theorem 2.7. Let $\mathcal{F}: X \rightarrow \mathbb{R}$ be a $C^{1}$ functional satisfying the Palais-Smale condition. If $\mathcal{F}$ has a pair of local minima or maxima, then $\mathcal{F}$ admits a third critical point.

\section{Proof of Theorem 1.1}

Let us introduce the following functionals $\Phi, \Psi: \mathbb{X}_{T}^{s} \rightarrow \mathbb{R}$ by

$$
\Phi(v):=\frac{1}{2}\|v\|_{\mathbb{X}_{T}^{s}}^{2}, \quad \Psi(v):=\varkappa_{s} \int_{\partial^{0} \mathcal{S}_{T}} \alpha(x) F(\operatorname{Tr}(v)) d x \quad \text { for all } v \in \mathbb{X}_{T}^{s} .
$$

From now on, we assume that (1.2) holds. It is easy to deduce that $\Phi$ is a coercive, continuously Gâteaux-differentiable and sequentially weakly lower semicontinuous functional. On the other hand, $\Psi$ is well-defined, continuously Gâteauxdifferentiable and also weakly continuous in $\mathbb{X}_{T}^{s}$ by virtue of (1.2).

By standard arguments the differentials of $\Phi$ and $\Psi$ are given by

$$
\Phi^{\prime}(v)(\varphi)=\iint_{\mathcal{S}_{T}} y^{1-2 s}\left(\nabla v \nabla \varphi+m^{2} v \varphi\right) d x d y
$$

and

$$
\Psi^{\prime}(v)(\varphi)=\varkappa_{s} \int_{\partial^{0} \mathcal{S}_{T}} \alpha(x) f(\operatorname{Tr}(v)) \operatorname{Tr}(\varphi) d x
$$

for every $\varphi \in \mathbb{X}_{T}^{s}$.

Next we establish a precise interval of values of the parameter $\lambda$ for which the functional $\mathcal{E}_{\lambda}$ defined in (2.6) admits at least three critical points. 
Theorem 3.1. Let $\alpha_{0}$ as in (1.1) and $\beta_{1}, \beta_{2}$ as in (1.6). Then, for every $\gamma>0$ and every $\lambda<\mu_{2}$, where

$$
\mu_{2}:=\frac{\gamma}{\alpha_{0}\left(a_{1} \beta_{1}+a_{2} \beta_{2} \gamma^{q-1}\right)}
$$

there exists a local minimum $v_{1}^{\lambda} \in \Phi^{-1}\left(\left(-\infty, \gamma^{2}\right)\right)$ of functional $\mathcal{E}_{\lambda}$ in $\mathbb{X}_{T}^{s}$.

Proof. Firstly, we estimate

$$
\varphi\left(\gamma^{2}\right):=\inf _{w \in \Phi^{-1}\left(\left(-\infty, \gamma^{2}\right)\right)} \frac{\left(\sup _{z \in \Phi^{-1}\left(\left(-\infty, \gamma^{2}\right)\right)} \Psi(z)\right)-\Psi(w)}{\gamma^{2}-\Phi(w)} .
$$

Let us consider the function

$$
\chi(r):=\frac{\sup _{z \in \Phi^{-1}((-\infty, r])} \Psi(z)}{r},
$$

with $r \in(0,+\infty)$.

Setting $F(t)=\int_{0}^{t} f(r) d r$, by (1.2) we get that

$$
F(t) \leq a_{1}|t|+\frac{a_{2}}{q}|t|^{q} \quad \text { for all } t \in \mathbb{R} .
$$

Then, (2.2) and (3.5) imply that, for all $z \in \mathbb{X}_{T}^{s}$, we have that

$$
\begin{aligned}
\Psi(z) & =\varkappa_{s} \int_{\partial^{0} \mathcal{S}_{T}} \alpha(x) F(\operatorname{Tr}(z)) d x \\
& \leq\left(\varkappa_{s} a_{1}|\operatorname{Tr}(z)|_{L^{1}(0, T)^{N}}+\varkappa_{s} \frac{a_{2}}{q}|\operatorname{Tr}(z)|_{L^{q}(0, T)^{N}}^{q}\right)|\alpha|_{L^{\infty}(0, T)^{N}} \\
& \leq\left(\varkappa_{s} a_{1} c_{1}\|z\|_{\mathbb{X}_{T}^{s}}+\varkappa_{s} \frac{a_{2}}{q} c_{q}^{q}\|z\|_{\mathbb{X}_{T}^{s}}^{q}\right)|\alpha|_{L^{\infty}(0, T)^{N}}
\end{aligned}
$$

which yields

(3.6) $\sup _{z \in \Phi^{-1}((-\infty, r])} \Psi(z) \leq \varkappa_{s} \sqrt{2 r} a_{1} c_{1}|\alpha|_{L^{\infty}(0, T)^{N}}+\varkappa_{s} \frac{(2 r)^{q / 2} a_{2} c_{q}^{q}}{q}|\alpha|_{L^{\infty}(0, T)^{N}}$.

Taking into account (3.4) and (3.6), we obtain that for any $r>0$ it holds

$$
\chi(r) \leq \varkappa_{s} \sqrt{\frac{2}{r}} a_{1} c_{1}|\alpha|_{L^{\infty}(0, T)^{N}}+\varkappa_{s} \frac{2^{q / 2} a_{2} c_{q}^{q}}{q} r^{q / 2-1}|\alpha|_{L^{\infty}(0, T)^{N}} .
$$

Hence, using (3.3) and (3.10), we conclude that

$$
\chi\left(\gamma^{2}\right)=\frac{\sup _{z \in \Phi^{-1}\left(\left(-\infty, \gamma^{2}\right]\right)} \Psi(z)}{\gamma^{2}}
$$




$$
\begin{aligned}
& \leq \varkappa_{s} \sqrt{2} \frac{a_{1} c_{1}}{\gamma}|\alpha|_{L^{\infty}(0, T)^{N}}+\varkappa_{s} \frac{2^{q / 2} a_{2} c_{q}^{q}}{q} \gamma^{q-2}|\alpha|_{L^{\infty}(0, T)^{N}} \\
& =\alpha_{0}\left(a_{1} \frac{\beta_{1}}{\gamma}+a_{2} \beta_{2} \gamma^{q-2}\right) \\
& =\frac{1}{\mu_{2}}
\end{aligned}
$$

Since $0 \in \Phi^{-1}\left(\left(-\infty, \gamma^{2}\right)\right)$ and $\Phi(0)=\Psi(0)=0$, we infer that

$$
\varphi\left(\gamma^{2}\right) \leq \chi\left(\gamma^{2}\right)
$$

Therefore, by Theorem 2.6, we deduce that for any $\lambda \in\left(0, \mu_{2}\right) \subseteq\left(0,1 / \varphi\left(\gamma^{2}\right)\right)$, there exists $v_{1}^{\lambda} \in \Phi^{-1}\left(\left(-\infty, \gamma^{2}\right)\right)$ such that

$$
\mathcal{E}_{\lambda}^{\prime}\left(v_{1}^{\lambda}\right)=\Phi^{\prime}\left(v_{1}^{\lambda}\right)-\lambda \Psi^{\prime}\left(v_{1}^{\lambda}\right)=0 .
$$

Moreover, $v_{1}^{\lambda}$ is a global minimum of the restriction of $\mathcal{E}_{\lambda}$ to $\Phi^{-1}\left(\left(-\infty, \gamma^{2}\right)\right)$.

Now, we introduce suitable test functions in $\mathbb{X}_{T}^{s}$. Let us set for $\varrho>0$ such that $F(\varrho)>0$ :

$$
w^{\varrho}(x, y):=\theta(m y) \varrho \text { for all }(x, y) \in \mathcal{S}_{T},
$$

where $\theta$ is as in (2.3). Clearly $w^{\varrho} \in \mathbb{X}_{T}^{s}$ and by using the fact that

$$
\int_{0}^{+\infty} y^{1-2 s}\left(\left|\theta^{\prime}(m y)\right|^{2}+m^{2}|\theta(m y)|^{2}\right) d y=m^{2 s} \varkappa_{s}
$$

we deduce

$$
\begin{aligned}
\left\|w^{\varrho}\right\|_{\mathbb{X}_{T}^{s}}^{2} & =\iint_{\mathcal{S}_{T}} y^{1-2 s}\left(\left|\nabla w^{\varrho}\right|^{2}+m^{2} w^{\varrho 2}\right) d x d y \\
& =T^{N} \int_{0}^{+\infty} y^{1-2 s}\left(\left|\theta^{\prime}(m y)\right|^{2}+m^{2}|\theta(m y)|^{2}\right) d y \\
& =\varkappa_{s} m^{2 s} \varrho^{2} T^{N}
\end{aligned}
$$

Next, we prove the following useful result.

Proposition 3.2. Let $\alpha_{0}$ as in (1.1), and $\varrho>0$ such that $F(\varrho)>0$. Further, let $w^{\varrho}$ be as in (3.7) and $\gamma>0$. Then, the following inequality holds

$$
\Phi\left(w^{\varrho}\right)>\gamma^{2}
$$

Moreover, setting

$$
\mu_{1}:=\frac{m^{2 s}}{2 \alpha_{0}} \frac{\varrho^{2}}{F(\varrho)}
$$


if $\mu_{1}<\mu_{2}$ and $\lambda \in\left(\mu_{1}, \mu_{2}\right)$, we have

$$
\Phi\left(w^{\varrho}\right)-\lambda \Psi\left(w^{\varrho}\right)<\gamma^{2}-\lambda \sup _{w \in \Phi^{-1}\left(\left(-\infty, \gamma^{2}\right]\right)} \Psi(w) .
$$

Proof. By (1.5) and (3.8) it follows that

$$
\Phi\left(w^{\varrho}\right)=\frac{1}{2}\left\|w^{\rho}\right\|_{\mathbb{X}_{T}^{s}}^{2}=\frac{1}{2} \varkappa_{s} m^{2 s} \varrho^{2} T^{N}>\gamma^{2},
$$

that is (3.9) is true. By (1.1), since $\operatorname{Tr}\left(w^{\varrho}\right)=\varrho$ and (1.4) implies that $F(\varrho)>0$, we get

$$
\int_{\partial^{0} \mathcal{S}_{T}} \alpha(x) F\left(\operatorname{Tr}\left(w^{\varrho}\right)\right) d x \geq \alpha_{0} T^{N} F(\varrho) .
$$

Then, (3.8), (3.12) and (3.10), yield

$$
\frac{\Psi\left(w^{\varrho}\right)}{\Phi\left(w_{\tau}^{\varrho}\right)} \geq \frac{2 \alpha_{0}}{m^{2 s}} \frac{F(\varrho)}{\varrho^{2}}=\frac{1}{\mu_{1}},
$$

which together with $\lambda \in\left(\mu_{1}, \mu_{2}\right)$ gives

$$
\chi\left(\gamma^{2}\right) \leq \frac{1}{\mu_{2}}<\frac{1}{\lambda}<\frac{1}{\mu_{1}} \leq \frac{\Psi\left(w^{\varrho}\right)}{\Phi\left(w^{\varrho}\right)} .
$$

As a consequence

$$
\begin{aligned}
\frac{\Psi\left(w^{\varrho}\right)-\sup _{w \in \Phi^{-1}\left(\left(-\infty, \gamma^{2}\right]\right)} \Psi(w)}{\Phi\left(w^{\varrho}\right)-\gamma^{2}} & \geq \frac{\Psi\left(w^{\varrho}\right)-\gamma^{2} \frac{\Psi\left(w^{\varrho}\right)}{\Phi\left(w^{\varrho}\right)}}{\Phi\left(w^{\varrho}\right)-\gamma^{2}} \\
& =\frac{\Psi\left(w^{\varrho}\right)}{\Phi\left(w^{\varrho}\right)} \\
& \geq \frac{1}{\mu_{1}}>\frac{1}{\lambda}
\end{aligned}
$$

that is (3.11) holds.

Now we define the following truncated functionals on $\mathbb{X}_{T}^{s}$

$$
\mathcal{E}_{\lambda}^{(\gamma)}(v):= \begin{cases}\gamma^{2}-\lambda \Psi(v) & \text { if } v \in \Phi^{-1}\left(\left(-\infty, \gamma^{2}\right]\right) \\ \mathcal{E}_{\lambda}(v) & \text { if } v \notin \Phi^{-1}\left(\left(-\infty, \gamma^{2}\right]\right)\end{cases}
$$

Fixed $\lambda>0$, it is easy to see that $\mathcal{E}_{\lambda}^{(\gamma)}$ is sequentially weakly lower semicontinuous. If $\mathcal{E}_{\lambda}^{(\gamma)}$ is also coercive on the Hilbert space $\mathbb{X}_{T}^{s}$, then it admits a global minimum $v_{2}^{\lambda} \in \mathbb{X}_{T}^{s}$, that is:

$$
\mathcal{E}_{\lambda}^{(\gamma)}\left(v_{2}^{\lambda}\right) \leq \mathcal{E}_{\lambda}^{(\gamma)}(v) \quad \text { for all } v \in \mathbb{X}_{T}^{s}
$$


Theorem 3.3. Let $\lambda \in\left(\mu_{1}, \mu_{2}\right)$ and assume that (1.7) holds. Then

$$
v_{2}^{\lambda} \notin \Phi^{-1}\left(\left(-\infty, \gamma^{2}\right]\right)
$$

and $\mathcal{E}_{\lambda}^{\prime}\left(v_{2}^{\lambda}\right)=0$, that is $v_{2}^{\lambda} \in \mathbb{X}_{T}^{s}$ is a critical point of $\mathcal{E}_{\lambda}$.

Proof. Let us assume by contradiction that

$$
v_{2}^{\lambda} \in \Phi^{-1}\left(\left(-\infty, \gamma^{2}\right]\right) .
$$

By (3.13) and the definition of $\mathcal{E}_{\lambda}^{(\gamma)}$, we infer that

$$
\gamma^{2}-\lambda \Psi\left(v_{2}^{\lambda}\right) \leq \mathcal{E}_{\lambda}\left(w^{\varrho}\right) .
$$

On the other hand, in view of Proposition 3.2, we know that

$$
\mathcal{E}_{\lambda}\left(w^{\varrho}\right)<\gamma^{2}-\lambda \Psi\left(v_{2}^{\lambda}\right),
$$

so we get a contradiction.

In previous result we have shown that for $\lambda \in\left(\mu_{1}, \mu_{2}\right)$ the support of $v_{2}^{\lambda}$ is not contained in the ball $B(0, \sqrt{2} \lambda)$.

We end this section giving the proof of our main result.

Proof of Theorem 1.1. By using (1.4), (3.10) and (3.3) it follows that $\mu_{1}<\mu_{2}$. Now, let us take $\lambda \in\left(\mu_{1}, \mu_{2}\right)$. By applying Theorems 3.1 and 3.3, we obtain the existence of two solutions $v_{1}^{\lambda}$ and $v_{2}^{\lambda}$ to (2.4). Since $v_{1}^{\lambda}$ and $v_{2}^{\lambda}$ are local minima, we can obtain the existence of a third solution to (2.4) via Theorem 2.7, provided that $\mathcal{E}_{\lambda}$ satisfies the Palais-Smale condition. Let us consider for a $c \in \mathbb{R}$ a sequence $\left(v_{n}\right)_{n} \subset \mathbb{X}_{T}^{s}$ such that

$$
\begin{gathered}
\mathcal{E}_{\lambda}\left(v_{n}\right) \longrightarrow c \text { and }\left\|\mathcal{E}_{\lambda}^{\prime}\left(v_{n}\right)\right\|_{\mathbb{X}_{T}^{s}} \longrightarrow 0 \quad \text { as } n \longrightarrow+\infty, \\
\left\|\mathcal{E}_{\lambda}^{\prime}\left(v_{n}\right)\right\|_{\mathbb{X}_{T}^{s}}:=\sup \left\{\left|\left\langle\mathcal{E}_{\lambda}^{\prime}\left(v_{n}\right), \varphi\right\rangle\right|: \varphi \in \mathbb{X}_{T}^{s} \quad \text { and }\|\varphi\|_{\mathbb{X}_{T}^{s}}=1\right\} .
\end{gathered}
$$

Recall that assumption (1.7) is satisfied by a $\delta \in(0,2)$, hence by using the Hölder's inequality we have that

$$
\int_{\partial^{0} \mathcal{S}_{T}}|\operatorname{Tr}(v)|^{\delta} d x \leq\left|\partial^{0} \mathcal{S}_{T}\right|^{\frac{2-\delta}{2}}|\operatorname{Tr}(v)|_{L^{2}(0, T)^{N}}^{\delta} \quad \text { for all } v \in \mathbb{X}_{T}^{s} ;
$$

this and (2.2) imply that

$$
\int_{\partial^{0} \mathcal{S}_{T}}|\operatorname{Tr}(v)(x)|^{\delta} d x \leq c_{2}^{\delta}\left|\partial^{0} \mathcal{S}_{T}\right|^{\frac{2-\delta}{2}}\|v\|_{\mathbb{X}_{T}^{s}}^{\delta} \quad \text { for all } v \in \mathbb{X}_{T}^{s} .
$$


Now, by (1.7) and (3.14) we obtain that

$$
\begin{aligned}
& \mathcal{E}_{\lambda}(v) \geq \frac{1}{2}\|v\|_{\mathbb{X}_{T}^{s}}^{2}-\varkappa_{s} \lambda M c_{2}^{\delta}|\alpha|_{L^{\infty}(0, T)^{N}}\left|\partial^{0} \mathcal{S}_{T}\right|^{\frac{2-\delta}{2}}\|v\|_{\mathbb{X}_{T}^{s}}^{\delta}-\varkappa_{s} \lambda M|\alpha|_{L^{\infty}(0, T)^{N}}\left|\partial^{0} \mathcal{S}_{T}\right| \\
& \quad \text { on } \mathbb{X}_{T}^{s} .
\end{aligned}
$$

As a consequence, $\mathcal{E}_{\lambda}$ is bounded from below and coercive; plainly the coercivity implies the boundedness of $\left(v_{n}\right)_{n}$ in $\mathbb{X}_{T}^{s}$.

Since $\mathbb{X}_{T}^{s}$ is reflexive, we can extract a subsequence, still denoted by $\left(v_{n}\right)_{n}$, such that $v_{n} \rightarrow v_{\infty}$ in $\mathbb{X}_{T}^{s}$ for some $v_{\infty}$, that is, for any $\varphi \in \mathbb{X}_{T}^{s}$ it holds

$$
\lim _{n \rightarrow+\infty} \iint_{\mathcal{S}_{T}} y^{1-2 s}\left(\nabla v_{n} \nabla \varphi+m^{2} v_{n} \varphi\right) d x d y=\iint_{\mathcal{S}_{T}} y^{1-2 s}\left(\nabla v_{\infty} \nabla \varphi+m^{2} v_{\infty} \varphi\right) d x d y
$$

Our aim to verify that indeed $\left(v_{n}\right)_{n}$ strongly converges to $v_{\infty}$ as $n \rightarrow+\infty$. Observe that by (3.1) and (3.2) it results that

$$
\left\langle\Phi^{\prime}\left(v_{n}\right), v_{n}-v_{\infty}\right\rangle=\left\langle\mathcal{E}_{\lambda}^{\prime}\left(v_{n}\right), v_{n}-v_{\infty}\right\rangle+\varkappa_{s} \lambda \int_{\partial^{0} \mathcal{S}_{T}} \alpha(x) f\left(\operatorname{Tr}\left(v_{n}\right)\right) \operatorname{Tr}\left(v_{n}-v_{\infty}\right) d x .
$$

By using $\left\|\mathcal{E}_{\lambda}^{\prime}\left(v_{n}\right)\right\|_{\mathbb{X}_{T}^{s}} \rightarrow 0$ and the fact that the sequence $\left(v_{n}-v_{\infty}\right)_{n}$ is bounded in $\mathbb{X}_{T}^{s}$, we infer that

$$
\lim _{n \rightarrow+\infty}\left\langle\mathcal{E}_{\lambda}^{\prime}\left(v_{n}\right), v_{n}-v_{\infty}\right\rangle=0 .
$$

On the other hand by (1.2) and Hölder inequality we have that

$$
\begin{aligned}
\int_{\partial^{0} \mathcal{S}_{T}} \alpha(x)\left|f\left(\operatorname{Tr}\left(v_{n}\right)\right)\right|\left|\operatorname{Tr}\left(v_{n}-v_{\infty}\right)\right| d x \\
\leq a_{1}|\alpha|_{L^{\infty}(0, T)^{N}} \int_{\partial^{0} \mathcal{S}_{T}}\left|\operatorname{Tr}\left(v_{n}-v_{\infty}\right)\right| d x \\
\quad+a_{2}|\alpha|_{L^{\infty}(0, T)^{N}} \int_{\partial^{0} \mathcal{S}_{T}}\left|\operatorname{Tr}\left(v_{n}\right)\right|^{q-1}\left|\operatorname{Tr}\left(v_{n}-v_{\infty}\right)\right| d x \\
\leq a_{1}|\alpha|_{L^{\infty}(0, T)^{N}}\left|\operatorname{Tr}\left(v_{n}-v_{\infty}\right)\right|_{L^{1}(0, T)^{N}} \\
\quad+a_{2}|\alpha|_{L^{\infty}(0, T)^{N}}\left|\operatorname{Tr}\left(v_{n}\right)\right|_{L^{q}(0, T)^{N}}^{q-1}\left|\operatorname{Tr}\left(v_{n}-v_{\infty}\right)\right|_{L^{q}(0, T)^{N}}
\end{aligned}
$$

By Theorem 2.2 it follows that

$$
\lim _{n \rightarrow+\infty} \int_{\partial^{0} \mathcal{S}_{T}} \alpha(x)\left|f\left(\operatorname{Tr}\left(v_{n}\right)\right)\right|\left|\operatorname{Tr}\left(v_{n}-v_{\infty}\right)\right| d x \longrightarrow 0,
$$

therefore by (3.17) and (3.16) we deduce that

$$
\lim _{n \rightarrow+\infty}\left\langle\Phi^{\prime}\left(v_{n}\right), v_{n}-v_{\infty}\right\rangle=0,
$$


that is

$\lim _{n \rightarrow+\infty} \iint_{\mathcal{S}_{T}} y^{1-2 s}\left(\left|\nabla v_{n}\right|^{2}+m^{2} v_{n}^{2}\right) d x d y-\iint_{\mathcal{S}_{T}} y^{1-2 s}\left(\nabla v_{n} \nabla v_{\infty}+m^{2} v_{n} v_{\infty}\right) d x d y=0$.

Hence, (3.15) and (3.18), yield

$$
\lim _{n \rightarrow+\infty} \iint_{\mathcal{S}_{T}} y^{1-2 s}\left(\left|\nabla v_{n}\right|^{2}+m^{2} v_{n}^{2}\right) d x d y=\iint_{\mathcal{S}_{T}} y^{1-2 s}\left(\left|\nabla v_{\infty}\right|^{2}+m^{2} v_{\infty}^{2}\right) d x d y
$$

Then, being $\mathbb{X}_{T}^{s}$ a Hilbert space, we get that

$$
\left\|v_{n}-v_{\infty}\right\|_{\mathbb{X}_{T}^{s}}^{2}=\left\|v_{n}\right\|_{\mathbb{X}_{T}^{s}}^{2}+\left\|v_{\infty}\right\|_{\mathbb{X}_{T}^{s}}^{2}-2\left\langle v_{n}, v_{\infty}\right\rangle_{\mathbb{X}_{T}^{s}} \longrightarrow 0 \quad \text { as } n \longrightarrow+\infty
$$

that is $v_{n} \rightarrow v_{\infty}$ strongly in $\mathbb{X}_{T}^{s}$. Finally, by (1.2) and the boundedness of $\alpha \in$ $L^{\infty}(0, T)^{N}$, by adapting [2, Theorem 9] we infer that $u_{i}^{\lambda}:=\operatorname{Tr}\left(v_{i}^{\lambda}\right) \in L^{\infty}(0, T)^{N}$ for $i \in\{1,2,3\}$.

Nevertheless, for further references, we prefer to give proof of this regularity in all details in next lemma.

Lemma 3.4. Let $v \in \mathbb{X}_{T}^{s}$ be a weak solution to (2.4). Then $\operatorname{Tr}(v) \in L^{\infty}(0, T)^{N}$.

Proof. Since $v$ is a weak solution to (2.4), equality (2.5) holds. Let us define $w:=v v_{K}^{2 \beta} \in \mathbb{X}_{T}^{s}$, where $v_{K}:=\min \{|v|, K\}, K>1$ and $\beta \geq 0$.

Pick $\varphi=w$ in $(2.5)$, so we get

$$
\begin{gathered}
\iint_{\mathcal{S}_{T}} y^{1-2 s} v_{K}^{2 \beta}\left(|\nabla v|^{2}+m^{2} v^{2}\right) d x d y+\iint_{D_{K}} 2 \beta y^{1-2 s} v_{K}^{2 \beta}|\nabla v|^{2} d x d y \\
=\varkappa_{s} \lambda \int_{\partial^{0} \mathcal{S}_{T}} \alpha(x) f(x, \operatorname{Tr}(v)) \operatorname{Tr}(v) \operatorname{Tr}\left(v_{K}\right)^{2 \beta} d x
\end{gathered}
$$

where $D_{K}:=\left\{(x, y) \in \mathcal{S}_{T}:|v(x, y)| \leq K\right\}$.

Then, by (3.19) and Theorem 2.1 we deduce that

$$
\begin{aligned}
& c_{2_{s}^{*}}^{-2}\left|\operatorname{Tr}(v) \operatorname{Tr}\left(v_{K}\right)^{\beta}\right|_{L^{2 *}(0, T)^{N}}^{2} \\
& \quad \leq\left\|v v_{K}^{\beta}\right\|_{\mathbb{X}_{T}^{s}}^{2}=\iint_{\mathcal{S}_{T}} y^{1-2 s}\left(\left|\nabla\left(v v_{K}^{\beta}\right)\right|^{2}+m^{2} v^{2} v_{K}^{2 \beta}\right) d x d y \\
& \quad=\iint_{\mathcal{S}_{T}} y^{1-2 s} v_{K}^{2 \beta}\left(|\nabla v|^{2}+m^{2} v^{2}\right) d x d y+\iint_{D_{K}} 2 \beta\left(1+\frac{\beta}{2}\right) y^{1-2 s} v_{K}^{2 \beta}|\nabla v|^{2} d x d y \\
& \quad \leq C_{\beta}\left[\iint_{\mathcal{S}_{T}} y^{1-2 s} v_{K}^{2 \beta}\left(|\nabla v|^{2}+m^{2} v^{2}\right) d x d y+\iint_{D_{K}} 2 \beta y^{1-2 s} v_{K}^{2 \beta}|\nabla v|^{2} d x d y\right]
\end{aligned}
$$




$$
=\widetilde{C}_{\beta} \int_{\partial^{0} \mathcal{S}_{T}} \alpha(x) f(x, \operatorname{Tr}(v)) \operatorname{Tr}(v) \operatorname{Tr}\left(v_{K}\right)^{2 \beta} d x
$$

where

$$
\widetilde{C}_{\beta}:=\varkappa_{s} \lambda\left(1+\frac{\beta}{2}\right) .
$$

By assumptions (1.1) and (1.2) we infer that

$$
\begin{aligned}
& \alpha(x) f(x, \operatorname{Tr}(v)) \operatorname{Tr}(v) \operatorname{Tr}\left(v_{K}\right)^{2 \beta} \\
& \quad \leq|\alpha|_{L^{\infty}(0, T)^{N}}\left[h(x)(1+|\operatorname{Tr}(v)|)^{2} \operatorname{Tr}\left(v_{K}\right)^{2 \beta}\right] \text { on } \partial^{0} \mathcal{S}_{T},
\end{aligned}
$$

where

$$
h(x):=\frac{|f(x, \operatorname{Tr}(v))|}{1+|\operatorname{Tr}(v)|} \leq C\left(1+|\operatorname{Tr}(v)|^{q-2}\right) \in L^{\frac{N}{2 s}}(0, T)^{N},
$$

for some $C>0$. Taking into account (3.20) and (3.21) we have that

$$
\left|\operatorname{Tr}(v) \operatorname{Tr}\left(v_{K}\right)^{\beta}\right|_{L^{2 *}(0, T)^{N}}^{2} \leq c_{2_{s}^{*}}^{2} \widetilde{C}_{\beta}|\alpha|_{L^{\infty}(0, T)^{N}} \int_{\partial^{0} \mathcal{S}_{T}}\left[h(x)(1+|\operatorname{Tr}(v)|)^{2} \operatorname{Tr}\left(v_{K}\right)^{2 \beta}\right] d x .
$$

Assume that $|\operatorname{Tr}(v)|^{\beta+1} \in L^{2}(0, T)^{N}$ for some $\beta \geq 0$. Fix $R>0$ and let $A_{1}=\{h \leq$ $R\}$ and $A_{2}=\{h>R\}$. Then

$$
\begin{aligned}
& \int_{\partial^{0} \mathcal{S}_{T}} h|\operatorname{Tr}(v)|^{2}\left|\operatorname{Tr}\left(v_{K}\right)\right|^{2 \beta} d x \\
& \quad \leq\left.\left. R|| \operatorname{Tr}(v)\right|^{\beta+1}\right|_{L^{2}(0, T)^{N}} ^{2}+\varepsilon(R)\left(\int_{\partial^{0} \mathcal{S}_{T}}\left|\operatorname{Tr}(v) \operatorname{Tr}\left(v_{K}\right)^{\beta}\right|^{2_{s}^{*}} d x\right)^{2 / 2_{s}^{*}}
\end{aligned}
$$

where $\varepsilon(R):=\left(\int_{A_{2}} h^{N / 2 s} d x\right)^{\frac{2 s}{N}} \rightarrow 0$ as $R \rightarrow \infty$.

In similar way, we can deal with the term $\int_{\partial^{0} \mathcal{S}_{T}} h(x)\left|\operatorname{Tr}\left(v_{K}\right)\right|^{2 \beta} d x$. Therefore, in view of (3.22) and (3.23), and choosing $R$ sufficiently large, we can see that

$$
\left|\operatorname{Tr}(v) \operatorname{Tr}\left(v_{K}\right)^{\beta}\right|_{L^{2 *}(0, T)^{N}}^{2} \leq C(1+R),
$$

for some $C>0$ independent of $K$.

Taking the limit as $K \rightarrow \infty$, we obtain $|\operatorname{Tr}(v)|^{\beta+1} \in L^{2_{s}^{*}}(0, T)^{N}$. This conclusion followed simply from assuming $|\operatorname{Tr}(v)|^{\beta+1} \in L^{2}(0, T)^{N}$.

Hence, by iterating $\beta_{0}=0$ and $\beta_{i}+1=\left(\beta_{i-1}+1\right) \frac{N}{N-2 s}$ if $i \geq 1$ in (3.24), we can infer that $\operatorname{Tr}(v) \in L^{q}(0, T)^{N}$ for all $q \in[2,+\infty)$. Since $\operatorname{Tr}(v)$ is a weak solution to $\left(P_{\lambda}\right)$, we deduce that $\left(-\Delta+m^{2}\right)^{s} \operatorname{Tr}(v) \in L^{p}(0, T)^{N}$ for any $p<+\infty$, and by using the embeddings for Bessel spaces [5], we deduce that $\operatorname{Tr}(v) \in C^{0, \alpha}\left([0, T]^{N}\right)$, for some $\alpha \in(0,1)$. 
In conclusion, we present a direct application of the main result of this work.

Example 3.5. Let $\alpha: \mathbb{R}^{N} \rightarrow \mathbb{R}$ be the $T$-periodic function defined as

$$
\alpha(x)=N+1+\sum_{i=1}^{N} \sin \left(\frac{2 \pi}{T} x_{i}\right) .
$$

We note that $\alpha$ is a continuous positive function such that $\alpha_{0}:=\min _{[0, T]^{N}} \alpha(x)=1$, that is $\alpha$ verifies (1.1). Now, take $q \in\left(2,2_{s}^{*}\right), \delta \in[1,2)$ and define

$$
\nu:=\max \left\{1, \sqrt{\frac{2}{\varkappa_{s}}} \frac{1}{m^{s} T^{N / 2}},\left[\frac{m^{2 s}}{2}\left(\beta_{1}+\beta_{2}\right)\right]^{\frac{1}{q-2}} q^{\frac{1}{q-2}}\right\} .
$$

Let $\varrho$ be a positive constant such that $\varrho>\nu$ and consider the continuous and positive function $f: \mathbb{R} \rightarrow \mathbb{R}$ defined as follows:

$$
f(t):= \begin{cases}1+|t|^{q-1} & \text { if } t \leq \varrho \\ 1+\varrho^{q-\delta} t^{\delta-1} & \text { if } t>\varrho\end{cases}
$$

It is clear that $|f(t)| \leq\left(1+|t|^{q-1}\right)$ for every $t \in \mathbb{R}$, and then (1.2) is fulfilled. Moreover, for every $t \in \mathbb{R}$, one has

$$
F(t) \leq\left(\varrho+\frac{\varrho^{q}}{\delta}\right)\left(1+|t|^{\delta}\right)
$$

Hence, hypothesis (1.7) is satisfied. On the other hand, condition (1.3) trivially holds and, since $\varrho>\nu$, one has

$$
\frac{F(\varrho)}{\varrho^{2}}=\frac{\int_{0}^{\varrho} f(\tau) d \tau}{\varrho^{2}}=\frac{\varrho^{q-2}}{q}+\frac{1}{\varrho}>\frac{\nu^{q-2}}{q}>\frac{m^{2 s}}{2}\left(\beta_{1}+\beta_{2}\right),
$$

and $\varrho>\sqrt{\frac{2}{\varkappa_{s}}} \frac{1}{m^{s} T^{N / 2}}$, i.e. conditions (1.4) and (1.5) are verified taking $\gamma=1$.

Therefore, all the assumptions of Theorem 1.1 are satisfied, hence, for every

$$
\lambda \in\left(\frac{m^{2 s}}{2} \frac{\varrho^{2}}{F(\varrho)}, \frac{1}{\beta_{1}+\beta_{2}}\right)
$$

the following problem

$$
\begin{cases}\left(-\Delta+m^{2}\right)^{s} u=\lambda \alpha(x) f(u) & \text { in }(0, T)^{N} \\ u\left(x+T e_{i}\right)=u(x) & \text { for all } x \in \mathbb{R}^{N}, i=1, \ldots, N,\end{cases}
$$

admits at least three weak solutions in $L^{\infty}(0, T)^{N} \cap \mathbb{H}_{T}^{s}$.

Acknowledgements. The authors would like to express their sincere gratitude to the anonymous referee for careful reading the manuscript and valuable comments and suggestions. The paper has been carried out under the auspices of the INdAM - GNAMPA Project 2017 titled: Teoria e modelli per problemi non locali. 


\section{References}

1. Ambrosio, V., Periodic solutions for a pseudo-relativistic Schrödinger equation, Nonlinear Anal. 120 (2015), 262-284. MR3348058

2. Ambrosio, V., Periodic solutions for the non-local operator pseudo-relativistic $\left(-\Delta+m^{2}\right)^{s}-m^{2 s}$ with $m \geq 0$, Topol. Methods Nonlinear Anal. 49 (2017), 75104. MR3635638

3. Ambrosio, V., Periodic solutions for critical fractional equations, Calc. Var. Partial Differential Equations 57 (2018), 45. 57 pp. MR3766988

4. Ambrosio, V. and Molica Bisci, G., Periodic solutions for nonlocal fractional equations, Commun. Pure Appl. Anal. 16 (2017), 331-344. MR3583529

5. Aronszajn, N. and Smith, K. T., Theory of Bessel potentials. I, Ann. Inst. Fourier (Grenoble) 11 (1961), 385-475. MR0143935

6. Barrios, B., Colorado, E., De Pablo, A. and Sánchez, U., On some critical problems for the fractional Laplacian operator, J. Differential Equations 252 (2012), 6133-6162. MR2911424

7. Brändle, C., Colorado, E., de Pablo, A. and Sánchez, U., A concave-convex elliptic problem involving the fractional Laplacian, Proc. Roy. Soc. Edinburgh Sect. A 143 (2013), 39-71. MR3023003

8. CAbré, X. and Sire, Y., Nonlinear Equations for fractional Laplacians I: Regularity, maximum principles, and Hamiltonian estimates, Ann. Inst. H. Poincaré Anal. Non Linéaire 31 (2014), 23-53. MR3165278

9. Caffarelli, L. and Silvestre, L., An extension problem related to the fractional Laplacian, Comm. Partial Differential Equations 32 (2007), 12451260. MR2354493

10. Carmona, R., Masters, W. C. and Simon, B., Relativistic Schrödinger operators: Asymptotic behavior of the eigenfunctions, J. Funct. Anal. 91 (1990), 117142. MR1054115

11. Di Nezza, E., Palatucci, G. and Valdinoci, E., Hitchhiker's guide to the fractional Sobolev spaces, Bull. Sci. Math. 136 (2012), 521-573. MR2944369

12. Dipierro, S., Medina, M. and Valdinoci, E., Fractional elliptic problems with critical growth in the whole of $\mathbb{R}^{n}$, Appunti. Scuola Normale Superiore di Pisa (Nuova Serie) [Lecture Notes. Scuola Normale Superiore di Pisa (New Series)] 15, Edizioni della Normale, Pisa, 2017, viii+152 pp. MR3617721

13. Lieb, E. H. and Loss, M., Analysis, 2nd ed., American Mathematical Society, Providence, RI, 2001. MR1817225

14. Molica Bisci, G., Rădulescu, V. and Servadei, R., Variational Methods for Nonlocal Fractional Problems. With a Foreword by Jean Mawhin, Encyclopedia of Mathematics and its Applications 162, Cambridge University Press, Cambridge, 2016. MR3445279

15. Pucci, P. and Serrin, J., Extensions of the mountain pass theorem, J. Funct. Anal. 59 (1984), 185-210. MR0766489

16. Rabinowitz, P. H., Minimax methods in critical point theory with applications to differential equations, CBMS Reg. Conf. Ser. Math. 65, American Mathematical Society, Providence, RI, 1986. MR0845785

17. RiCCERI, B., A general variational principle and some of its applications, J. Comput. 
Appl. Math. 113 (2000), 401-410. MR1735837

18. Ryznar, M., Estimate of Green function for relativistic $\alpha$-stable processes, Potential Anal. 17 (2002), 1-23. MR1906405

Vincenzo Ambrosio

Dipartimento di Ingegneria Industriale e Scienze Matematiche

Università Politecnica delle Marche

Via Brecce Bianche, 12

60131 Ancona

Italy

ambrosio@dipmat.univpm.it

Rossella Bartolo

Dipartimento di Meccanica, Matematica e Management

Politecnico di Bari

Via Orabona, 4

70125 Bari

Italy

rossella.bartolo@poliba.it

Received November 13, 2018

in revised form April 10, 2019
Giovanni Molica Bisci

Dipartimento di Scienze Pure e Applicate (DiSPeA)

Università degli Studi di Urbino Carlo Bo Piazza della Repubblica 13

61029 Urbino

Italy

giovanni.molicabisci@uniurb.it 